\title{
Simulation numérique d'un écoulement de plasma en aval d'une sphère non équipotentielle
}

\author{
A. Soubeyran ( $\left.{ }^{1}\right)$, E. Coggiola $\left({ }^{2}\right)$ and J. J. Berthelier $\left({ }^{3}\right)$ \\ (1) Département d'Etude et de Recherche en Technologie Spatiale, Centre d'Etude et de Recherche de \\ Toulouse, 2 av. E. Belin, 31055 Toulouse Cedex, France \\ (2) \# 245, Petrie Science Building, Physics Department, North York, Ontario/M3J 1P3, Canada \\ (3) Centre de Recherche en Physique de l'Environnement (CNET/CNRS), 4 av. de Neptune, 94107 Saint- \\ Maur-des-Fossés, France
}

(Reçu le 10 mai 1989, révisé le 18 décembre 1989, accepté le 12 janvier 1990)

\begin{abstract}
Résumé. - Nous présentons un code numérique permettant de simuler l'effet d'une charge négative localisée à l'arrière d'un satellite en orbite basse dans l'ionosphère, sur la structure du sillage de celui-ci. Nous montrons que cette charge ne peut être neutralisée par les ions du plasma ambiant et que, dans ce cas, le sillage ne peut généralement pas être représenté par un simple modèle d'expansion thermique.
\end{abstract}

\begin{abstract}
We present a new code which can be used to simulate the effect of a negative charge located at the rear of a spacecraft in low ionospheric orbit on its wake structure. We show that this charge cannot be neutralized by the ionospheric ions and that, in this case, the wake cannot be represented by a simple model of thermal expansion.
\end{abstract}

\section{Introduction.}

En raison des nombreuses anomalies de fonctionnement apparues sur les satellites géostationnaires, l'interaction de ces derniers avec le plasma magnétosphérique a fait l'objet d'investigations poussées, qui permettent aujourd'hui de bien comprendre les phénomènes de charge et de claquage à l'origine de ces anomalies [6,13, 15, 16, 17]. En orbite basse, bien qu'aucun phénomène de ce type n'ait été rapporté avec certitude, il apparaît cependant prudent d'étudier les aspects spécifiques de l'interaction des satellites avec le plasma ambient, en particulier en orbite polaire. L'environnement ionisé d'un satellite est en effet fortement inhomogène, de sorte qu'une partie, électriquement isolée de la structure principale, peut acquérir un potentiel différent du potentiel flottant de l'ensemble, et être ainsi susceptible d'initier une décharge. Cette remarque s'applique en particulier au cas d'un spationaute lors d'activités extra-véhiculaires [9].

Plusieurs satellites instrumentés, dont la navette spatiale, ont déjà permis de détecter un sillage, ainsi que d'autres perturbations du plasma environnant
$[14,22]$. De plus, certains auteurs ont fait récemment état de niveaux d'électrisation de l'ordre du kilovolt négatif sur un satellite DMSP [8] et corrélé l'apparition de tels potentiels à la présence d'intenses précipitations aurorales d'électrons énergétiques, d'origine magnétosphérique [25]. Des expérimentations conduites récemment en chambre à plasma, confirment également l'existence de potentiels négatifs élevés $(\leqslant-1 \mathrm{kV})$ sur une métallisation flottante placée dans le sillage d'une maquette en ambiance ionosphérique et soumise à un flux d'électrons énergétiques [4].

Ces résultats inquiétants ont suscité de nombreux travaux théoriques, relatifs à l'interaction entre un satellite et le plasma ionosphérique $[11,12]$; la complexité du problème a cependant conduit les auteurs à diverses simplifications. Il convient ainsi de signaler qu'à l'exception des travaux de Parker $[19,20]$, seul le cas de structures équipotentielles a été envisagé $[2,10]$. Ayant constaté l'importance potentielle des phénomènes de charge différentielle en orbite basse (exemple : EVA), nous avons cherché à mieux les quantifier à l'aide d'un code numérique adapté. 


\section{Description du code.}

En raison de la difficulté du problème et de la capacité limitée des moyens de calcul, nous avons décidé de traiter des géométries simplifiées, bidimensionnelles, de satellites de petites tailles, de l'ordre du mètre.

La résolution a tout d'abord été menée à bien, dans le cas d'un cylindre conducteur infini, présentant une section électriquement isolée côté sillage [4]. Une forme sphérique paraissant cependant une meilleure approximation pour beaucoup de satellites, nous avons donc repris le code élaboré précédemment et l'avons modifié de façon à rendre compte d'une telle symétrie. Une calotte électriquement isolée, pouvant être chargée, est placée du côté aval de l'écoulement. C'est en effet dans le sillage proche que l'on s'attend à trouver des conditions physiques analogues à celles régnant en orbite géostationnaire, propices à l'apparition de fortes charges. Sa dimension est choisie suffisamment petite pour que son bord soit éloigné de la frontière du sillage.

\subsection{NOTATIONS.}

Satellite :

$\begin{array}{ll}R_{\text {sat }} & : \text { rayon du satellite } \\ V_{\text {sat }} & : \text { vitesse du satellite }\end{array}$

Plasma :

$N_{\mathrm{i}} \quad:$ densité ionique $\left(N_{0}\right.$ à l'infini $)$

$T_{\mathrm{i}} \quad:$ température ionique

$V_{\text {th. i }}:$ vitesse thermique des ions

$M_{\mathrm{i}}=V_{\text {sat }} / V_{\text {th. i }}:$ nombre de Mach ionique

$\omega_{\mathrm{c}} \quad:$ pulsation cyclotronique ionique

$r_{\mathrm{L}} \quad:$ rayon de Larmor ionique

$N_{\mathrm{e}} \quad$ : densité électronique ( $N_{0}$ à l'infini)

$T_{\mathrm{e}} \quad:$ température électronique

$V_{\text {th. e }} \quad:$ vitesse thermique électronique

$\lambda_{\text {Debye }} \quad:$ longueur de Debye

$E \quad$ : champ électrique

$\phi \quad$ : potentiel référencé par rapport au potentiel plasma

$B \quad$ : champ magnétique

$e \quad:$ charge élémentaire (positive)

$k \quad:$ constante de Boltzmann

2.2 HYPOTHÈSES SIMPLIFICATRICES. - Les caractéristiques du milieu ionosphérique (voir Tab. I), permettent certaines simplifications :

1) Plasma sans collisions dans le domaine d'interaction.

2) Plasma mésothermique :

- vitesse thermique ionique petite devant les autres vitesses :

$$
V_{\text {th. i }} \ll V_{\text {sat }} \ll V_{\text {th. } \mathrm{e}}
$$

Tableau I. - Paramètres ionosphériques autour de $500 \mathrm{~km}$ d'altitude [1]. Le plasma est beaucoup plus froid et dense que celui de l'orbite géostationnaire.

[Ionospheric parameters at $500 \mathrm{~km}$ altitude. The plasma is colder and denser than the one at geostationary orbit.]

Température des électrons $(\approx$ ions $) \quad: 1000 \mathrm{~K} \approx 0,1 \mathrm{eV}$

Vitesse thermique des électrons $\quad: 2 \times 10^{5} \mathrm{~m} . \mathrm{s}^{-1}$

Vitesse thermique des ions $\left(\mathrm{O}^{+}\right): 10^{3} \mathrm{~m} \cdot \mathrm{s}^{-1}$

Vitesse d'un satellite

Densité électronique

Longueur de Debye

$10^{3} \mathrm{~m} \cdot \mathrm{s}^{-1}$
$8 \times 10^{3} \mathrm{~m} \cdot \mathrm{s}^{-1}$

$10^{10} \mathrm{~m}^{-3}$

$2 \times 10^{-2} \mathrm{~m}$

$: 10^{4} \mathrm{~m}$

$3 \times 10^{-2} \mathrm{~m}$

Rayon cyclotronique des électrons

Rayon cyclotronique des ions $\left(\mathrm{O}^{+}\right)$

: $5 \mathrm{~m}$

- distribution de Maxwell-Boltzmann pour les électrons :

$$
N_{\mathrm{e}}=N_{0} \cdot \exp \left(e \cdot \phi / k \cdot T_{\mathrm{e}}\right) .
$$

3) Une seule espèce d'ions $\left(\mathrm{O}^{+}\right.$en orbite basse, $\mathrm{Ar}^{+}$en chambre expérimentale), dérivants par rapport au satellite. Nous ne cherchons pas à prendre en compte l'existence de populations d'ions lents autour des grandes structures spatiales (dégazage, moteurs d'appoints...), ou dans les grandes chambres à plasma (échange de charge avec les neutres résiduels). Notre étude se limite ainsi aux cas de petits satellites, peu susceptibles d'engendrer de tels effets.

4) Pas de champ magnétique :

Afin de ne pas alourdir exagérément les calculs, nous avons décidé de ne pas tenir compte du champ magnétique terrestre. Les résultats restent valables dans la réalité, dans les limites précisées ci-dessous.

- Déviation des ions.

L'influence du champ magnétique sur le mouvement ionique ne sera sensible que pour des satellites de taille supérieure au rayon de giration des ions thermiques. En effet, si l'on note $\Delta t$ la durée pendant laquelle un ion peut être en interaction avec un satellite :

$$
\Delta t \approx \frac{R_{\mathrm{sat}}}{V_{\text {th. } \mathrm{i}}} .
$$

L'écart $\Delta Y$ entre la trajectoire rectiligne théorique suivant $X$ (sans champ magnétique) et la trajectoire hélicoïdale réelle, n'apparaîtra que pour :

$$
\begin{gathered}
\frac{\Delta Y}{\Delta X}=\frac{\frac{V_{\text {th. i }}}{\omega_{\mathrm{c}}} \times\left(1-\cos \left(\omega_{\mathrm{c}} \times \Delta t\right)\right)}{V_{\text {sat }} \times \Delta t} \approx \\
\approx \frac{V_{\text {th. } \mathrm{i}} \times \omega_{\mathrm{c}} \times \Delta t}{2 \times V_{\text {sat }}}>0,1 \\
\Rightarrow \omega_{\mathrm{c}} \times \Delta t=\frac{R_{\text {sat }}}{r_{\mathrm{L}}}>0,2 \times M_{\mathrm{i}} \approx 1,6 .
\end{gathered}
$$


Ce rayon $r_{\mathrm{L}}$ ëtant d'environ 5 mètres dans l'ionosphère, nous limiterons notre étude à des satellites de dimension comparable.

Par ailleurs, sous l'influence des champs électriques apparaissant dans le sillage, le centre guide des trajectoires ioniques peut acquérir une vitesse de dérive notable. Cependant, une fois encore il faut tenir compte des échelles de temps. Si l'on note $\Delta X$ le déplacement d'un ion dans la direction du champ électrique et $\Delta Y$ son déplacement dans la direction $(E X B)$, pendant la durée $\Delta t$ :

$$
\begin{aligned}
\frac{\Delta Y}{\Delta X}=\frac{\frac{E}{B} \times\left(\frac{\sin \left(\omega_{\mathrm{c}} \times \Delta t\right)}{\omega_{\mathrm{c}}}-\Delta t\right)}{\frac{E}{B} \times \frac{1-\cos \left(\omega_{\mathrm{c}} \times \Delta t\right)}{\omega_{\mathrm{c}}}} & \approx \\
\approx \frac{\omega_{\mathrm{c}} \times \Delta t}{3} & =\frac{1}{3} \times \frac{R_{\text {sat }}}{r_{\mathrm{L}}} \approx \frac{R_{\text {sat }}}{15}
\end{aligned}
$$

(en supposant le champ électrique perpendiculaire au champ magnétique).

Ainsi, l'écart entre la trajectoire réelle et la trajectoire simulée ne sera important $(\Delta Y / \Delta X>0,1)$ que pour des satellites de diamètre supérieur à trois mètres, que nous ne chercherons donc pas à modéliser.

- Champ électrique induit.

En raison des limitations précédentes sur la taille des structures, la force électromotrice dipôlaire induite est de l'ordre du potentiel flottant $\left(E \leqslant V_{\text {sat }} X . B \approx 0.3 \mathrm{~V} / \mathrm{m}\right)$, et décroît en $1 / r^{2}$, beaucoup plus vite que ce dernier. Compte tenu de plus de l'effet d'écran dû à la gaine électronique, il n'y a pas à en tenir compte au-delà de quelques longueurs de Debye. Ceci est encore plus vrai en présence de potentiels différentiels bien plus élevés, appliqués à l'arrière du satellite.

5) Nous supposons également, comme cela a été fait très généralement pour l'étude des caractéristiques des sondes de Langmuir dans un plasma en mouvement qu'il n'y a pas d'ondes de choc en amont du satellite. Ceci se justifie aisément si l'on considère que les ions et les électrons qui atteignent la surface du satellite sont collectés par celle-ci et par conséquent qu'ils disparaissent du milieu : dans ce cas, le satellite ne représente pas un obstacle au mouvement des ions et il n'y a donc aucune raison pour qu'une perturbation soit engendrée en amont. L'hypothèse de collection des particules par la surface, sans émission secondaire, est tout à fait adéquate en raison de leur faible énergie au moment de l'impact.

6) Absence d'électrons émis par la surface chargée (secondaires, photoélectrons, etc.) :

Nous considérons la situation de "pire cas ", d'une surface à l'ombre, bombardée par des électrons énergiques $\left(E_{\mathrm{e}} \geqslant 10 \mathrm{keV}\right)$, pour lesquels le taux d'émission secondaire est très faible $(\tau \leqslant 0.1)$.
Rappelons par ailleurs, que la dimension caractéristique de cette surface est choisie suffisamment petite (environ un dixième du diamètre du satellite) pour l'isoler au maximum du plasma dérivant. Cet élément de surface du satellite, porté à un fort potentiel, ne pourrait ainsi se décharger que par un courant d'ions attirés dans le sillage. Coggiola [4] a déjà montré que ce courant devait être très faible pour un satellite cylindrique infiniment long, nous verrons qu'il en est de même dans le cas d'une sphère.

2.3 Algorithme (Fig. 1). - Nous résolvons itérativement le système Hamilton-Poisson, dans des conditions aux limites stationnaires. Les équations

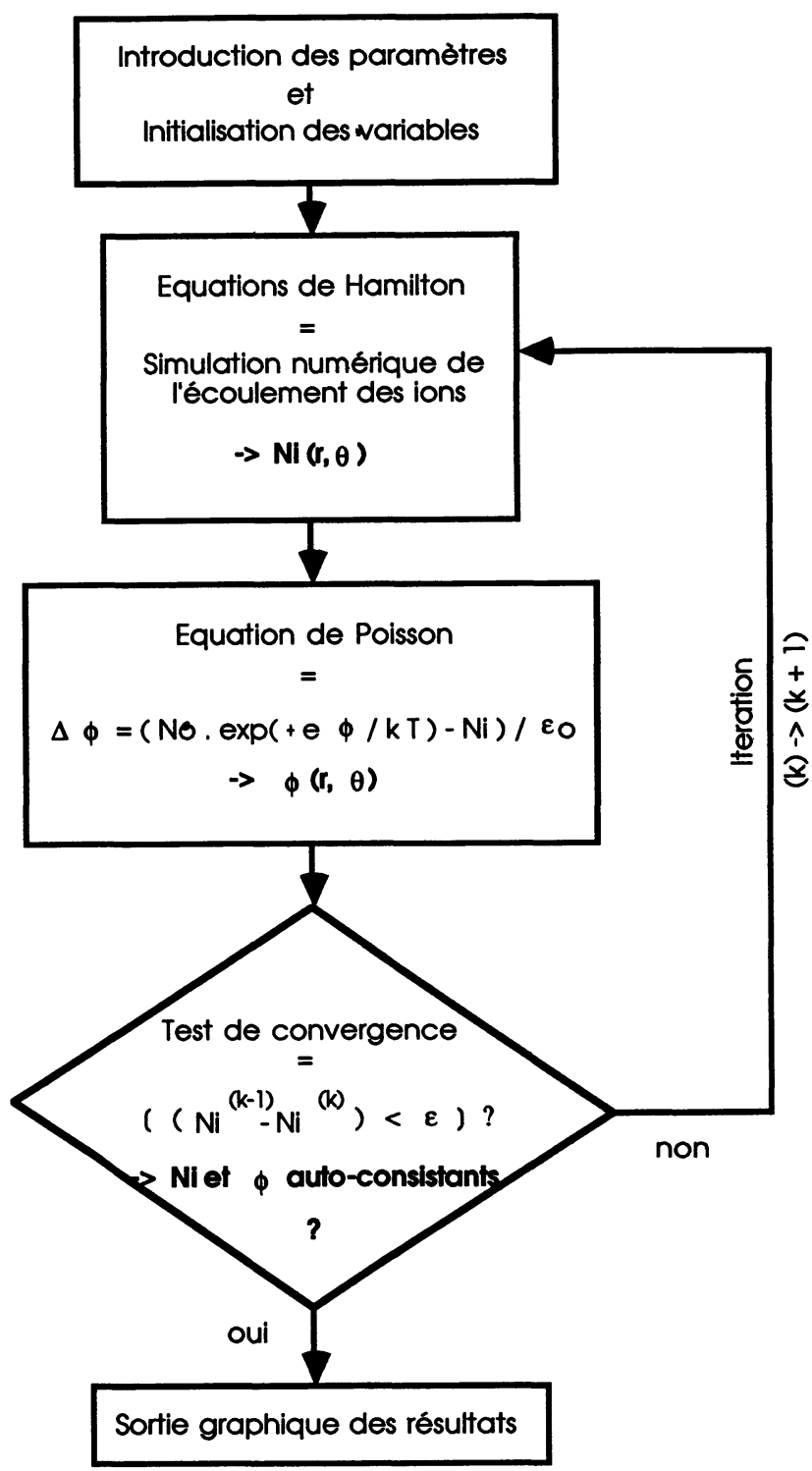

Fig. 1. - Algorithme de résolution numérique des équations couplées de Hamilton et Poisson (explications dans le texte).

[Algorithm for the numerical solution of the coupled Hamilton and Poisson equations.] 
de Hamilton, ou équations du mouvement, appliquées à un grand nombre de particules de simulation permettent, par leur intégration numérique, de connaître les trajectoires et vitesses moyennes des particules réelles. Elles conduisent en cela aux mêmes résultats que l'équation de Vlasov, utilisée parfois pour obtenir directement les fonctions de distribution [5].

\section{- Hamilton (Equations du mouvement).}

- Injection d'un « front » de particules de simulation ( $\equiv$ ions), à la vitesse $V_{\text {sat }}$ (éventuellement légèrement corrigée par une distribution maxwellienne de vitesses thermiques: $\left.V_{\text {thi }} \ll V_{\text {sat }}\right)$, par la surface d'entrée, perpendiculaire au déplacement du satellite ( $\theta=0$, voir Fig. 2).

- Intégration des équations de Hamilton, compte tenu des champs électriques connus aux nœuds du réseau (on choisit initialement un potentiel nul partout).

- Déposition pondérée de charges aux nœuds du réseau, jusqu'à la sortie hors du domaine de calcul de toutes les trajectoires (voir plus bas).

$\rightarrow$ distribution de densité ionique.

\section{- Poisson.}

- Calcul des potentiels à partir des densités en tout nœud du réseau.

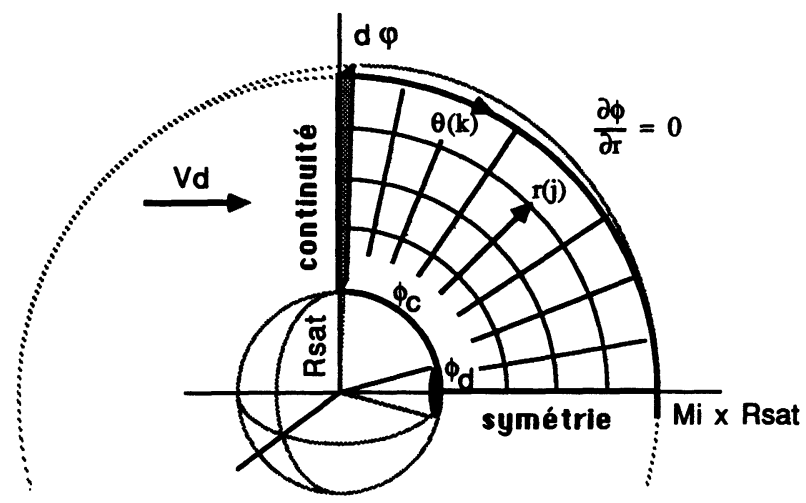

Fig. 2. - Représentation schématique de la zone d'interactions. La partie quadrillée correspond au domaine de calcul adopté, les conditions aux limites (en trait gras) et les coordonnées $(r, \theta)$ étant précisées. En grisé, la frontière d'entrée des particules. $V_{\mathrm{d}}$ : vitesse de dérive des ions ; $M_{\mathrm{i}}$ : nombre de Mach ionique ; $\phi_{\mathrm{c}}, \phi_{\mathrm{d}}$ : potentiels à la surface du satellite; $R_{\text {sat }}$ : rayon du satellite $; \varphi$ : coordonnée angulaire pour laquelle le système est invariant.

[Schematic representation of the interaction area; the hatched zone corresponds to the computational mesh, with indication of the coordinate system $(r, \theta)$ and of the boundary conditions. The particle entry frontier is shaded. $V_{\mathrm{d}}$ : drift velocity of ions; $M_{\mathrm{i}}$ : ionic Mach number; $\phi_{\mathrm{c}}, \phi_{\mathrm{d}}$ : satellite surface potentials ; $\boldsymbol{R}_{\text {sat }}$ : satellite radius ; $\varphi$ : invariant angular coordinate.] $\rightarrow$ distribution de potentiel $(\Rightarrow$ champ électrique, et densité électronique).

Ce schéma est répété jusqu'à la convergence du système. Celle-ci est réalisée lorsque la distribution de densité conduit, par l'équation de Poisson, à une distribution de potentiel, qui redonne, après intégration des équations de Hamilton et déposition de charges, cette même distribution de densité (solution auto-consistante).

2.4 Domaine de Calcul (Fig. 2). - (N.B. : les angles sont comptés dans le sens inverse du sens trigonométrique, l'axe vertical étant pris pour origine).

1) Par suite de la symétrie axiale du système, on s'intéresse au demi-espace $-\pi / 2 \leqslant \theta \leqslant \pi / 2$.

2) L'écoulement ionique est supposé peu perturbé en amont (Sect. 2.2.5 et [23]). Ainsi, le champ électrique apparaissant au voisinage de la surface du satellite reste confiné sur une épaisseur maximum de quelques longueurs de Debye. Comme ce champ dérive du potentiel flottant, il ne saurait de toute façon communiquer aux ions, qu'une énergie de l'ordre de grandeur de leur énergie thermique, très inférieure à leur énergie de dérive par rapport au satellite. Nous limiterons donc le domaine d'étude aux angles : $0 \leqslant \theta \leqslant \pi / 2$.

3) Le rayon du contour externe est choisi, dans la mesure du possible (capacité mémoire), à Mi rayons satetlite de la surface: En effet, les études théoriques et expérimentales [7,21, 22] s'accordent à montrer que l'essentiel de la perturbation se trouve limité à cette zone.

4) Le rayon interne correspond à la surface du satellite.

\subsection{CONDITIONS AUX LIMITES.}

1) Condition de symétrie sur l'axe central (réflection spéculaire des particules).

2) Condition de continuité sur l'axe vertical d'entrée des particules :

$$
\partial \phi / \partial \theta=0 .
$$

3) Condition de Neumann à l'« infini » :

$$
\partial \phi / \partial r=0 \text {. }
$$

4) Condition de Dirichlet à la surface du satellite : $\phi_{c}$ sur la partie principale $\phi_{\mathrm{d}}$ sur la calotte isolée.

2.6 Equations de Hamilton. - Par suite de l'invariance du système par rotation autour de l'axe du déplacement, les équations sont indépendantes de la variable angulaire correspondante. Le volume de simulation ressemble alors à un demi quartier d'orange, dont l'angle d'ouverture est arbitrairement 
petit (Fig. 2). Pour obtenir une densité de charge uniforme, en l'absence de perturbations, il est donc nécessaire d'introduire des particules identiques avec une répartition proportionnelle à leur distance à l'axe, ou bien d'attribuer à des particules distribuées uniformément, un « poids » (nombre équivalent de particules réelles) proportionnel à cette distance. Dans la mesure où les phénomènes intéressants se produisent au voisinage de l'axe et par suite des limitations de l'ordinateur, nous avons préféré la seconde solution qui évite de traiter beaucoup de trajectoires loin de l'axe, où leurs effets sont négligeables.

Pour toutes les particules, le système d'équations différentielles du premier ordre est ensuite résolu numériquement suivant une méthode de type Runge-Kutta-Gill d'ordre 4, avec un pas d'intégration $\delta$ t constant.

A chaque pas de temps, chaque particule dépose sa charge autour de sa nouvelle position. Cette charge est distribuée entre les quatre nœuds les plus proches, selon une méthode de répartition bilinéaire dite PIC (Particle In Cell) [3]. Lorsque le front de charge a atteint les limites du domaine de calcul (à « l'infini » ou sur la surface du satellite), la somme des charges accumulées en chaque nœud du réseau, le long de toutes les trajectoires, donne les densités ioniques en ces points, après une normalisation tenant compte en particulier des dimensions variables des cellules du réseau.

2.7 Equation de Poisson. - Nous utilisons les variables sans dimension suivantes :

$$
\begin{gathered}
\eta=-\frac{e \phi}{k T_{\mathrm{e}}}, \quad \zeta=\frac{r}{\lambda_{\text {Debye }}} \\
n_{\mathrm{i}}=\frac{N_{\mathrm{i}}}{N_{0}}, \quad n_{\mathrm{e}}=\frac{N_{\mathrm{e}}}{N_{0}}=\mathrm{e}^{-\eta} .
\end{gathered}
$$

L'équation de poisson s'écrit alors :

$$
\begin{aligned}
\frac{1}{\zeta^{2}} \times \frac{\partial}{\partial \zeta}\left(\zeta^{2} \frac{\partial \eta}{\partial \zeta}\right) & +\frac{1}{\zeta^{2} \times \cos \theta} \times \\
& \times \frac{\partial}{\partial \theta}\left(\cos \theta \frac{\partial \eta}{\partial \theta}\right)=n_{\mathrm{i}}-\mathrm{e}^{-\eta}
\end{aligned}
$$

Cette équation différentielle du second ordre non linéaire est mise sous forme discrète suivant la méthode des différences finies :

$\left((\delta \zeta, \delta \theta):\right.$ maillage régulier de l'espace $\left(\zeta_{j}\right.$, $\left.\theta_{k}\right)$, Fig. 2)

$$
\begin{aligned}
& {[\eta(j+1, k)-2 \times \eta(j, k)+} \\
& +\eta(j-1, k)] \times\left(\frac{1}{(\delta \zeta)^{2}}+\frac{1}{4 \times \zeta_{j}^{2}}\right) \\
& +[\eta(j+1, k)-\eta(j-1, k)] \times \frac{1}{\delta \zeta \times \zeta_{j}}
\end{aligned}
$$

$$
\begin{aligned}
& +[\eta(j, k+1)-2 \times \eta(j, k) \\
& +\eta(j, k-1)] \times\left(\frac{1}{(\delta \theta)^{2}}-\frac{1}{8}\right) \times \frac{1}{\zeta_{j}^{2}} \\
& -[\eta(j, k+1)-\eta(j, k-1)] \times \frac{\operatorname{tg}\left(\theta_{k}\right)}{2 \times \delta \theta} \times \frac{1}{\zeta_{j}^{2}} \\
& =n_{\mathrm{i}}(j, k)-\mathrm{e}^{-\eta(j, k)} .
\end{aligned}
$$

Ce système est résolu par une méthode itérative de type Gauss-Seidel [18].

\section{Résultats.}

Nous allons analyser ici les résultats obtenus pour deux configurations très différentes.

\subsection{SATEllite DE PETITE TAILle EN ORBITE BASSE. (Voir Tab. II).}

Nous avons tracé les isopotentielles et isodensités ioniques en aval de la sphère, pour trois potentiels de la calotte isolée (Fig. 3). Ces trois potentiels correspondent à trois états de charge plausibles de cette surface : non chargée (surface totale équipotentielle), chargée par une polarisation active (prochaine génération de panneaux polaires : quelques centaines de Volts) et chargée par des électrons de précipitation aurorale $\left(E_{\mathrm{e}} \geqslant 1 \mathrm{keV}\right)$. La surface conductrice principale est supposée au potentiel flottant, calculé par :

$$
\begin{aligned}
\phi_{\mathrm{f}} & =-\frac{k \times T(\mathrm{~K})}{2 \times e} \times \operatorname{Ln}\left[\frac{2 \times k \times T(\mathrm{~K})}{\pi \times m_{\mathrm{i}} \times V_{\text {sat }}^{2}}\right] \\
& =-0,36 \text { Volt }
\end{aligned}
$$

pour une sonde dans un plasma supersonique $\left(V_{\text {sat }}>V_{\text {th. i }}\right)[1]$.

Tableau II. - Caractéristiques moyennes d'un petit satellite et de son environnement en orbite basse -polaire. Le rayon du satellite est grand devant la longueur de Debye, à l'inverse des conditions de l'orbite géostationnaire.

[Average characteristics of small satellites and their environment in low Earth polar orbits. The satellite radius is larger than the Debye length, contrary to the geostationary orbit conditions.]

$$
\left.\begin{array}{l}
R_{\text {sat }}=1 \mathrm{~m} \\
T_{\mathrm{c}}=T_{\mathrm{i}}=0,15 \mathrm{eV} \\
m_{\mathrm{i}}=2,66 \times 10^{-26} \mathrm{~kg} \\
V_{\text {sat }}=8 \times 10^{3} \mathrm{~m} \cdot \mathrm{s}^{-1} \\
N_{0}=1 \times 10^{10} \mathrm{~m}^{-3} \\
\phi_{\mathrm{c}}=\phi_{\mathrm{f}}=-0,36 \mathrm{~V} \\
\phi_{\mathrm{d}}=-0,36,-100,-1000 \mathrm{~V}
\end{array}\right\}
$$



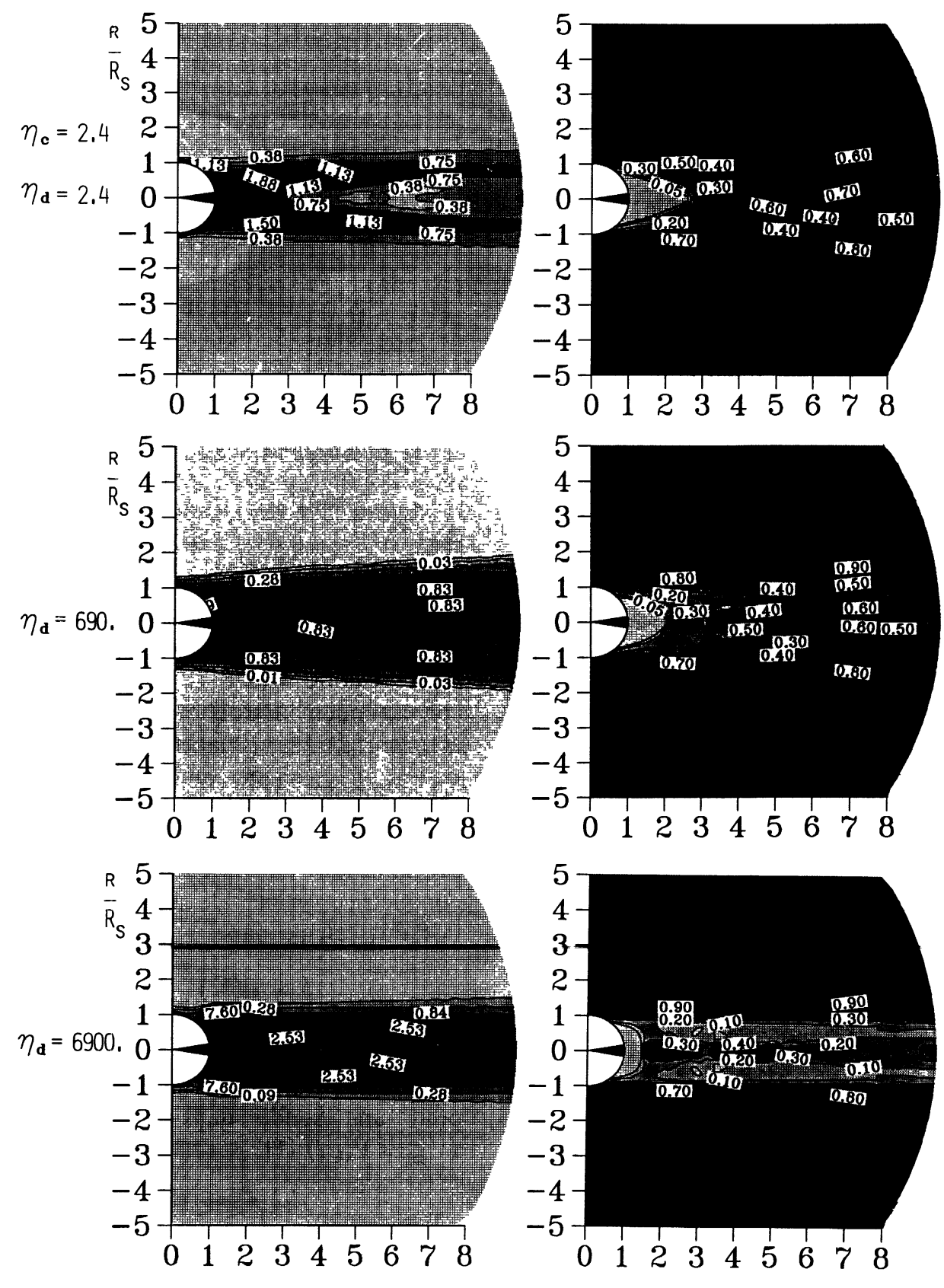

DISTRIBUTION DE POTENTIEL : $\eta$

DISTRIBUTION DE DENSITE IONIQUE : $\frac{N_{1}}{N_{0}}$

Fig. 3. - Structures de potentiel et de densité ionique pour trois polarisations d'une surface isolée dans le sillage d'un satellite en orbite basse. Le sillage se vide lorsque le potentiel différentiel augmente.

[Structure of the potential and ion density in the wake of a L.E.O. spacecraft for three values of the voltage applied to the insulated surface at the rear of the spacecraft. The ion density in the wake decreases if the voltage on the insulated surface increases.]

La structure la plus caractéristique est associée à une «focalisation» des ions le long de l'axe du système au-delà de $2-3 R_{\text {sat }}$, juste après la zone de raréfaction proche de la surface. Cette longueur est nettement plus courte que celle attendue pour des raisons purement cinétiques, de l'ordre de $M_{\mathrm{i}} \cdot R_{\mathrm{sat}}$, soit $8 R_{\text {sat }}$. Il est probable qu'elle résulte, d'une part du potentiel flottant non nul de la surface conductrice principale [24], d'autre part de la charge d'espace négative associée au sillage très proche.

On observe toujours un sillage très inhomogène, mais le code ne fournit qu'une solution stationnaire approchée qui ne permet pas de conclure quant à la réalité et l'ampleur de cette inhomogénéité. Dans le 
cas d'un satellite équipotentiel $\left(\phi_{\mathrm{c}}=\phi_{\mathrm{d}}\right)$, la zone de raréfaction est associée à un puits de potentiel, en accord avec les travaux théoriques de Al'Pert et al. [1], pour une sphère telle que $\zeta \gg 1$.

Les simulations numériques réalisées pour des polarisations de $-100 \mathrm{~V}$ et $-1000 \mathrm{~V}$ de la calotte isolée, montrent que, tout en attirant les ions, cette polarisation «vide » une partie du sillage. Cet effet, paradoxal au premier abord, s'explique ainsi : lorsque les ions, animés d'une vitesse de dérive importante, pénètrent dans la zone où se fait sentir le potentiel, ils sont accélérés vers l'axe, d'autant plus que le potentiel de la surface isolée est grand (Figs. 4a, b). Du fait que la perturbation pénètre

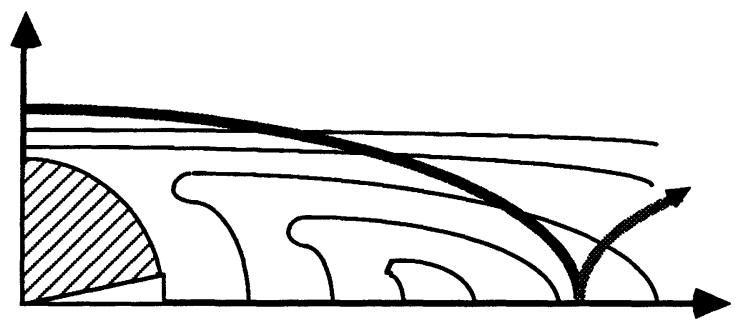

(a) Sans Polarisation

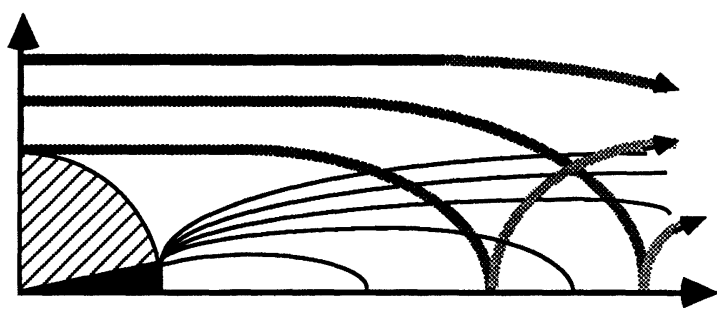

(b) Avec Polarisation ( $\zeta$ grand : satellite )

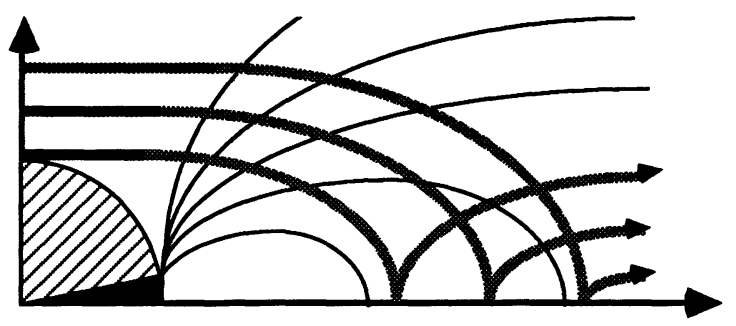

(c) Avec Polarisation ( $\zeta$ petit : Maquette)

\section{Equipotentielle}

\section{$\rightarrow$ Trajectoire ionique}

Fig. 4. - Trajectoires ioniques, en trait d'autant plus clair que la vitesse est grande, illustrant les déplacements de la zone de « focalisation » pour diverses structures de potentiel. Un grand satellite écrante la perturbation de potentiel dans son sillage, alors que celle-ci s'étend hors du sillage d'un petit satellite.

[Ion trajectories (the thickness of the lines decreases when the velocity increases) showing the focalisation effect for various potential structure. A large spacecraft shields the wake potential disturbance, while a small one does not.] peu dans le plasma environnant, leur flux varie peu en fonction du potentiel différentiel. Cependant, quand ce potentiel croît leur vitesse augmente, donc la densité ionique diminue. Sur l'axe, l'effet de focalisation compense cette accélération et la densité tend à augmenter localement.

Comme dans le cas d'un cylindre infini [4], on tirera donc les conclusions suivantes, pour une configuration telle que $\zeta \gg 1$, et un écoulement fortement supersonique (orbite basse, relativement faible densité) :

- En aval d'un satellite équipotentiel se forme un sillage quasiment vide d'ions, associé à une charge d'espace négative.

- L'apparition d'une surface polarisée négativement à l'arrière du satellite entraîne une extension de la zone de déplétion ionique, avec cependant une focalisation accrue des ions sur l'axe du sillage.

- Même pour de forts potentiels de la calotte isolée, la perturbation semble rester confinée dans le sillage « classique » $\left(M_{\mathrm{i}} \cdot R_{\mathrm{sat}}\right)$.

Par rapport au cas du cylindre, l'écoulement autour d'une sphère se distingue par la forte focalisation des ions au voisinage de l'axe, en particulier pour de forts potentiels différentiels. Cependant on n'observe toujours aucune collection d'ions sur la surface isolée côté sillage.

\subsection{Modèle DE SATEllite DANS UNE CHAMBRE EXPÉRIMENTALE. - (Voir Tab. III).}

Dans ce cas, la maquette est placée dans un plasma dérivant $\left(V_{\text {dériv }} \equiv V_{\text {sat }}\right)$ et l'on polarise activement une partie de sa surface, en aval de l'écoulement.

L'observation des isodensités ioniques montre une « focalisation » beaucoup moins prononcée des ions, vers l'axe central (Fig. 5). Cet allongement du sillage

Tableau III. - Caractéristiques moyennes d'une maquette de satellite et de son environnement, en chambre à plasma. Le plasma est plus dense et plus rapide que le plasma ionosphérique.

[Average characteristics of a mock-up and its environment for experiments in a plasma chamber. The plasma is denser and faster than the ionospheric one.]

$$
\begin{aligned}
& R_{\text {sat }}=0,1 \mathrm{~m} \\
& T_{\mathrm{e}}=T_{\mathrm{i}}=0,1 \mathrm{eV} \\
& m_{\mathrm{i}}=5,98 \times 10^{-26} \mathrm{~kg} \\
& \eta_{\mathrm{c}}=-\phi_{\mathrm{c}} / T(\mathrm{eV})=0 \\
& \eta_{\mathrm{d}}=-\phi_{\mathrm{d}} / T(\mathrm{eV})= \\
& =0,1000,10000 \\
& V_{\text {sat }}=15 \times 10^{3} \mathrm{~m} \cdot \mathrm{s}^{-1} \quad \zeta=R_{\text {sat }} / \lambda_{\text {Debye }}=13 \\
& N_{0}=1 \times 10^{11} \mathrm{~m}^{-3} . \quad M_{\mathrm{i}}=V_{\text {sat }} / V_{\text {th. } \mathrm{i}}=29 \\
& \phi_{\mathrm{c}}=0 \mathrm{~V} \\
& \phi_{\mathrm{d}}=0,-100,-1000 \mathrm{~V}
\end{aligned}
$$



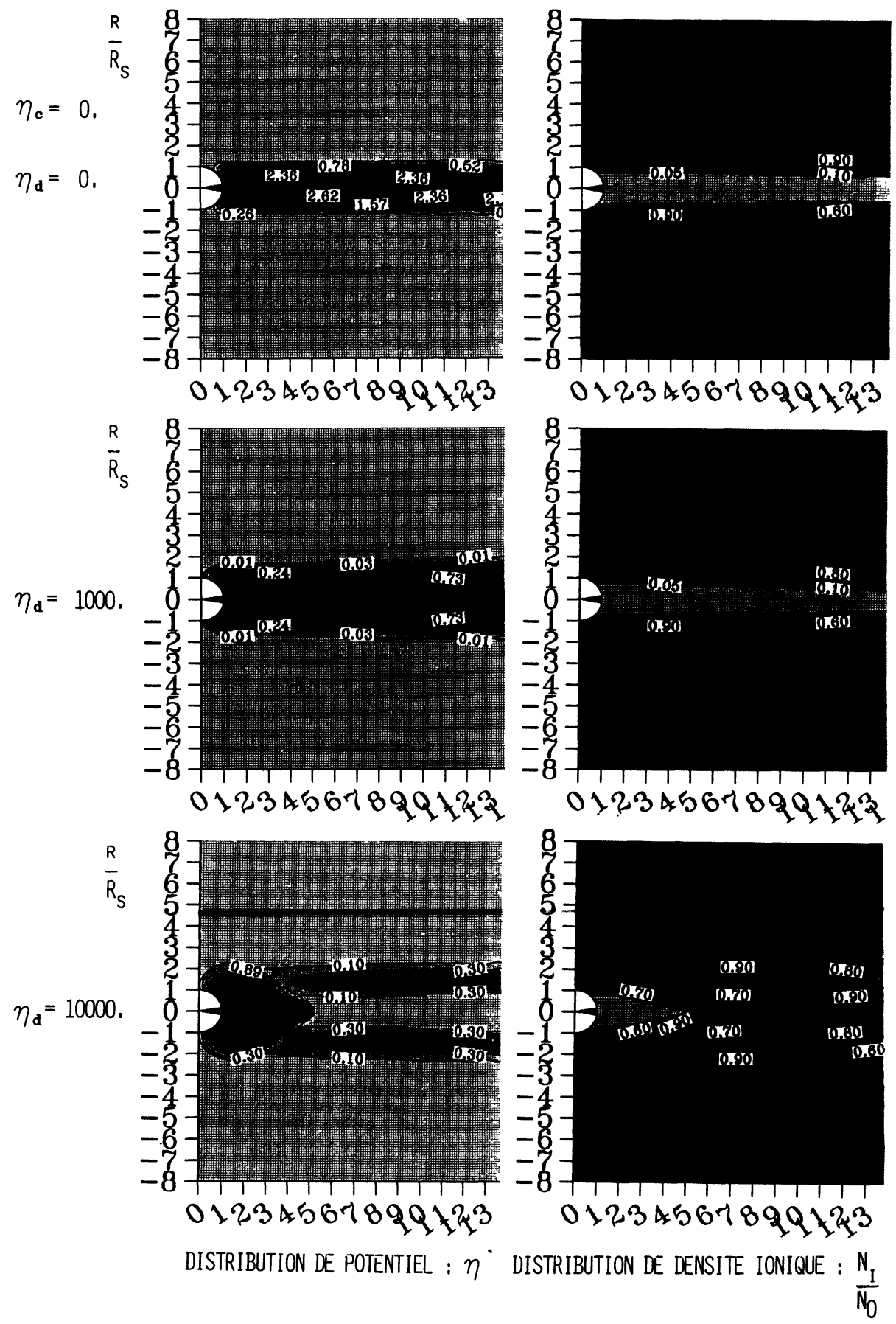

Fig. 5. - Structures de potentiel et de densité ionique pour trois polarisations d'une surface isolée dans le sillage d'une maquette en chambre à plasma. Le sillage se remplit lorsque le potentiel différentiel augmente.

[Potential and ion density wake structures of a mock-up in a plasma chamber, for the three values of the voltage applied to the insulated surface at the rear of the mock-up. The ion density in the wake increases with the voltage applied to the insulated surface.]

proche s'explique partiellement par un nombre de Mach ionique plus élevé, qui résulte en fait d'une vitesse de dérive plus grande, pour des ions plus lourds animés d'une plus faible agitation thermique, que pour un satellite en orbite basse. Dans un premier temps, pour alléger le calcul, nous ne tenions pas compte de cette agitation thermique dans le mouvement des ions (ions monocinétiques). L'extension du sillage est alors limitée seulement par l'effet du champ électrique provenant de la charge d'espace négative dans le sillage proche (puits de potentiel) qui est plus faible pour les ions $\mathrm{Ar}^{+}$que pour les ions $\mathrm{O}^{+}$. La simulation complète montre en fait que les effets cumulés du champ électrique et de 
l'agitation thermique, provoque encore une « fermeture » du sillage assez proche $\left(\approx 10 R_{\text {sat }}\right)$ (Fig. 6).

Contrairement à la configuration précédente (Sect. III.1), on constate maintenant que la densité ionique dans le sillage devient d'autant plus importante que la polarisation de la calotte isolée est élevée. Cette tendance inverse s'explique certainement par le fait que dans ce cas, le champ créé par la calotte polarisée ne reste plus confiné dans le sillage mais pénètre profondément dans le plasma. Les ions dérivants rencontrent encore un gradient de potentiel qui accroît leur vitesse, mais cette fois-ci leur flux augmente aussi avec l'intensité de la polarisation (Fig. 4c). Coggiola avait montré pour un cylindre, que l'extension de la perturbation de potentiel hors du sillage, est fortement dépendante du facteur sans dimension $\zeta$, et d'autant plus grande que $\zeta$ est petit [4]. C'est bien ce que nous constatons ici aussi, dans le cas d'une sphère.

Nous retiendrons de ce second cas les points suivants :

- en l'absence de polarisation de la surface isolée, on observe de nouveau un puits de potentiel dans le sillage proche, associé à une zone raréfiée assez étendue, si l'on ne tient pas compte de l'agitation thermique ionique. Mais l'effet de celle-ci n'est pas négligeable dans ce cas ;

- une forte polarisation de la calotte isolée conduit à une perturbation du potentiel, qui pénètre profondément dans le plasma, hors du sillage,

- dans ce cas, la densité des ions dans le sillage est d'autant plus élevée que la polarisation est forte. On remarquera que cette tendance est inversée par rapport au cas d'un satellite réel, en orbite basse.

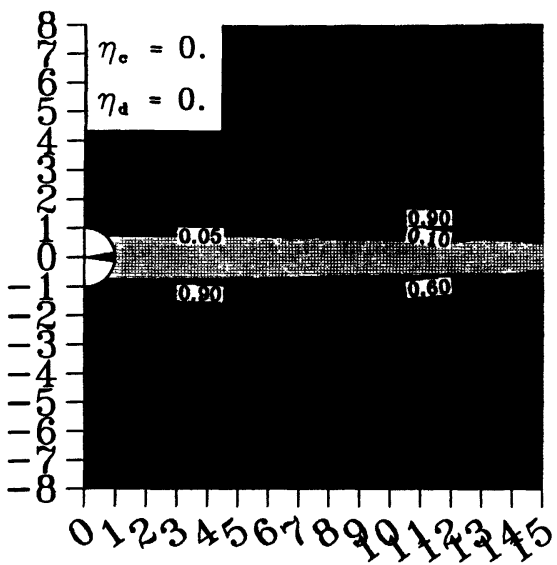

SANS MOUVEMENT THERMIQUE

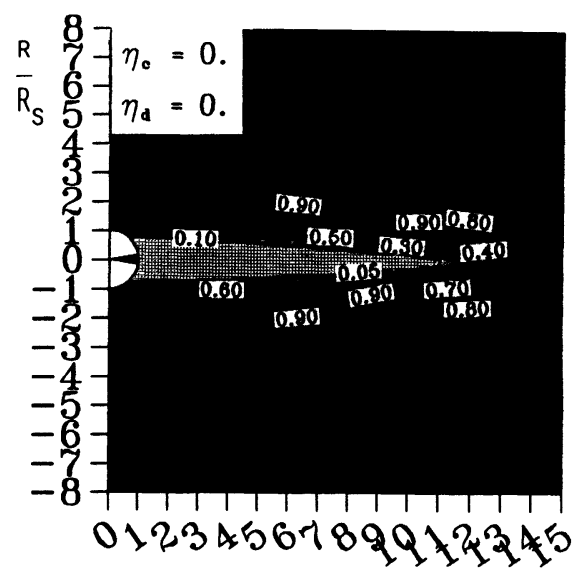

AVEC MOUVENENT THERMIQUE

Fig. 6. - Effet de l'agitation thermique sur la structure d'un sillage. L'expansion thermique joue un rôle important lorsqu'aucun potentiel n'est appliqué au satellite.

[Effect of the ion temperature on the structure of the wake. Thermal expansion plays a prominent part when the satellite is at plasma potential.]

\section{Conclusions.}

Le code que nous avons développé permet aujourd'hui de traiter numériquement un grand nombre de situations où un corps présente un mouvement relatif par rapport à un plasma. A l'aide des cas limites bidimensionnels du cylindre infini [4] et de la sphère, nous pensons pouvoir décrire la plupart des phénomènes liés au déplacement d'une structure réelle tridimensionnelle dans un plasma de type ionosphérique. L'environnement simulé est encore relativement simple (pas de champ magnétique, une seule population ionique, électrons traités implicitement, etc...), mais l'originalité de ce travail réside surtout dans la prise en compte d'une polarisation différentielle sur la face aval de la structure, là où elle est le plus susceptible d'apparaître. Les résultats obtenus pour une sphère confirment en effet les calculs réalisés antérieurement pour un cylindre :

Les ions dérivant ne peuvent donner lieu à aucun courant ionique, limitant la charge négative différentielle apparaissant sur une surface isolée en aval d'un satellite, lors de précipitations aurorales.

A travers deux exemples d'application très différents, nous espérons avoir montré toute la puissance et l'intérêt de ces nouvelles simulations numériques, qui permettent de mieux comprendre certains phénomènes physiques complexes, difficilement accessibles à l'expérience. Elles contribuent ainsi à une meilleure évaluation des risques spécifiques encourus par les systèmes placés en orbite basse, en particulier lors de conditions d'apparition de charges différentielles. 


\section{Bibliographie}

[1] Al'Pert Y. A., Gurevich A. V. and Pitaevskit L. P., Space Physics with Artificial Satellite (Consultant Bureau, New York) 1965.

[2] BARKer T. G., Analytic and Observational Approaches to Spacecraft Auroral Charging, A.F.G.L.TR-87-0021, S-CUBED, Scientific Report $N^{\circ} 1$ (Nov. 1986).

[3] Birdsall C. K. and Langdon A. B., Plasma Physics via Computer Simulation (McGraw-Hill, New York) 1985.

[4] Coggiola E., Etude Théorique et Expérimentale de l'Ecoulement de Plasma autour d'un Cylindre Non Equipotentiel, Thèse doctorat ENSAE, CERToulouse/DERTS (16 juin 1988).

[5] Delcroix J. L., Physique des plasmas I (Monographie Dunod, Paris) 1963.

[6] Fennel J. F., Koons H. C., Leung M. S. and MizerA P. F., The Aerospace Corporation. A Review of SCATHA Satellite results : Charging and Discharging, ESA-SP-198 (Noordjwik, The Netherlands) Sept. 1983.

[7] Gurevich A. V., Pitaevski L. P. and Smirnova V. V., Ionospheric Aerodynamics, Space Sci. Rev. 9 (1969) 805.

[8] Gussenhoven M. S., HaRdy D. A., Rich F., BURKE W. J. and YEH H.-C., High-Level Spacecraft Charging in the Low-Altitude Polar Auroral Environment, J. Geophys. Res. 90 (1985) 11009.

[9] Katz I., Mandell M. J., Jongeward G. A. and LILleY J. R. Jr., Astronaut Charging in the Wake of a Polar Orbiting Shuttle, AIAA-857035-CP, S-CUBED, AIAA Shuttle Environment And Operations II Meeting (Nov. 13-15, 1985).

[10] Katz I., Mandell M. J. and PaRKs D. E., Effect of object potentials on the wake of a flowing plasma, J. Appl. Phys. 62 (1987) 2675.

[11] LAframboise J. G., Is There a Good Way to Model Spacecraft Charging in the Presence of SpaceCharge Coupling, Flow, and Magnetic Fields, Proc. Air Force Geophys. Lab., Report $\mathrm{N}^{\circ}$ AFGL-TR-83-0046 (1983) p. 57.

[12] Laframboise J. G., Calculation of Escape Currents Emitted from Negatively Charged Spacecraft Surfaces in a Magnetic Field, J. Geophys. Res. 93 (1988) 1933.

[13] Levy L., Sarrail D., Philippon J. P., Catani J. P. and Fourouet J. M., Sur la Possibilité de Charge Différentielle de Plusieurs Kilovolts dans le Secteur Jour de l'Orbite Géosynchrone,
AGARD Conference on « The Aerospace Environment at High Altitudes and its Implications for Spacecraft Charging and Communication » (The Hague, The Netherlands) 2-6 June 1986, p. 17-1/12.

[14] Murphy G., Pickett J., D’Angelo N. and Kurth W. S., Measurements of Plasma Parameters in the vicinity of the Space Shuttle, Planetary and Space Sci. 34 (1986) 993.

[15] AFGL « Proc. of the Spacecraft Charging Technology Conf. ", AFGL-TR-77-0051 (1977).

[16] NASA « Spacecraft Charging Technology - 1978» NASA conf. publ. 2071, AFGL-TR-79-0082 (1978).

[17] NASA « Spacecraft Charging Technology - 1980 », NASA conf. publ. 2182, AFGL-TR-81-0270 (1980).

[18] Nougier J. P., Méthodes de calcul Numérique (Masson, Paris) 1983.

[19] PARKer L. W., Differential Charging and Sheath Asymetry of Non-Conducting Spacecraft Due to Plasma Flows, J. Geophys. Res. 83 (1978) 4873.

[20] PARKer L. W., Contributions to sheath and Wake Modelling, Eur. Space Agency Spec. Publ., ESA SP-198 (1983) p. 81.

[21] Samir U., Wildman P. J., Rich F., Brinton H. C. and SAGALYNR.C., About the Parametric Interplay Between Ionic Mach Number, Body Size, and Satellite Potential in Determining the Ion Depletion in the Wake ot the S3-2 Satellite, $J$. Geophys. Res. 86 (1981) 11161.

[22] SAMIR U., Stone N. H. and Wright K. H. Jr., On Plasma Disturbances Caused by the Motion of the Space Shuttle and Small Satellites: A Comparison of In Situ Observations, J. Geophys. Res. 91 (1986) 277.

[23] SAMIR U., The Interaction of Large Space Structures with the Near-Earth Environment, Proceedings of the Air Force Geophysics Laboratory Workshop on Natural Charging, Hanscom-AFB-MA, AFGL-TR-83-0046 (Jan. 25, 1983) p. 235.

[24] Stone N. H., The Plasma Wake of Mesosonic Conducting Bodies, Part 1, An Experimental Parametric Study of Ion Focusing by the Plasma Sheath, J. Plasma Phys. 25 (1981) 351.

[25] YeH H. C. and Gussenhoven S. M., The Statistical Environment for Defense Meteorological Satellite Program Eclipse Charging, J. Geophys. Res. 92 (1987) 7705. 